\title{
A Frequency-Tunable Metamaterial-Based Antenna Using a Reconfigurable AMC Groundplane
}

\author{
Marco Salucci ${ }^{1}$, Giacomo Oliveri ${ }^{1}$, Micah Gregory ${ }^{2}$, Douglas Werner ${ }^{2}$, Andrea Massa $^{1}$ \\ ${ }^{1}$ ELEDIA Research Center@DISI, University of Trento, Trento, Italy, andrea.massa@unitn.it \\ ${ }^{2}$ Comp. Electromag. and Antennas Research Lab (CEARL), The Pennsylvania State University, PA 16802, dhw@psu.edu
}

\begin{abstract}
The design of a low-profile narrowband antenna that is tunable over a wide frequency range is presented. A spline-based Ultra-Wideband (UWB) antenna is synthesized by means of a time-domain-based PSO and is mounted on top of a reconfigurable Artificial Magnetic Conductor (AMC) obtained by replicating a square unit cell in a $15 \times 15$ periodic lattice. The AMC is composed by a planar array of periodic metallic strips printed on top of a PEC-backed dielectric substrate, connected by tunable capacitors (varactors), and has been designed to work within the 1-4 [GHz] bandwidth. The reflection coefficient of the resulting narrowband antenna system can be easily controlled inside a wide frequency range by tuning the capacitance value $C$ of the varactors inside the AMC groundplane.
\end{abstract}

Index Terms-Tunable artificial magnetic conductor (AMC), spline-based UWB antenna, tunable antenna.

\section{INTRODUCTION}

In the last few years, there has been a growing interest in the design of Artificial Magnetic Conductors (AMC) and their integration inside advanced electromagnetic devices [1]-[5], as well as, more in general, in their application in the field of innovative devices based on metamaterials [6]. AMCs are Electromagnetic Bandgap (EBG) structures composed of planar periodic metallic elements printed on top of a thin PECbacked dielectric substrate and are designed to mimic, over a relatively narrow bandwidth, the behavior of a Perfect Magnetic Conductor (PMC). Unlike PEC ground planes, AMC structures can provide high surface impedance over a narrowband operating frequency range, reflecting EM waves in-phase with constructive interference and acting as Frequency Selective Surfaces (FSS). Due to this property, many researchers recently proposed the use of AMCs as ground planes for the design of low-profile antennas with reduced back-radiation, controlled broadside gain and tunable steering angle [7]-[9].

Within this context, reconfigurable AMCs have recently attracted a lot of attention, since the working frequency of these EBG structures can be easily tuned by locally modifying the control voltage of some lumped elements inserted between the patches and the ground plane [9] or between neighbor patches [10]. In [9] a phase discontinuity along the E-field direction is obtained by locally changing the polarization of tunable capacitors (varactor diodes) inserted between the FSS and the metallic ground plane, resulting in adaptive beam steering of the integrated antenna system. In [10], the

This work has been developed within the EMERALD Project funded by the Autonomous Province of Trento - Calls for proposal "Team 2011". reflection response of the AMC surface is electronically tuned by modifying the bias voltage of tunable capacitors inserted between the metallic patches. Such a design permits adaptively modifying the working frequency of the AMC, effectively controlling the reflection coefficient of a broadband opensleeve dipole mounted above it. Consequently, the radiating element turns out to be narrowband, while its resonant frequency can be shifted within the operational bandwidth of the AMC by acting on the bias voltage of its tunable capacitors [10].

Concerning the design of Ultra-Wideband (UWB) antennas, a great variety of approaches have been presented in the scientific literature for the synthesis of radiators characterized by compact size, low profile, as well as good impedance matching and good radiation properties over a significantly wide frequency range. In this regard, Evolutionary Algorithms (EAs) are clearly one of the most promising solution to deal with such a constrained design problem [12],[13]. In particular, the PSO-driven spline-based synthesis approach proposed in [14] represents an efficient and effective methodology for UWB antenna design thanks to its flexibility in dealing with heterogeneous user-defined requirements and to its high computational efficiency, as demonstrated in [15]-[21].

This work is aimed at assessing the performances of a frequency-tunable antenna system composed by a reconfigurable AMC groundplane coupled with a UltraWideband (UWB) radiator. In more detail, the UWB radiating element is described by spline curves and it is synthesized by means of a Particle Swarm Optimization (PSO) iterative process based on time-domain analysis which takes into account the radiation properties of the individual under test [22],[23]. Numerical simulations of the return loss of the integrated antenna system verify the wideband reconfigurability of the antenna impedance matching frequency by tuning the AMC capacitors.

\section{DESIGN PROCEDURE}

\section{A. Synthesis of the spline UWB element}

The time-domain PSO-based synthesis approach described in [22] is applied to design a UWB antenna having the largest possible bandwidth within the 1-4 [GHz] frequency range. More in detail, the antenna geometry is modeled by a radiating 
structure and a metallic groundplane printed onto an Arlon dielectric substrate $\left(\varepsilon_{r}=3.38\right)$ of thickness $0.78[\mathrm{~mm}]$. Both the radiating part and the groundplane are described with spline curves and are defined by a set of $K$ control points $\left\{P_{k}=\left(x_{p k}\right.\right.$, $\left.\left.z_{p k}\right) ; k=1, \ldots, K\right\}$ and $H$ control points $\left\{Q_{h}=\left(x_{q h}, z_{q h}\right) ; h=\right.$ $1, \ldots, H\}$, respectively.

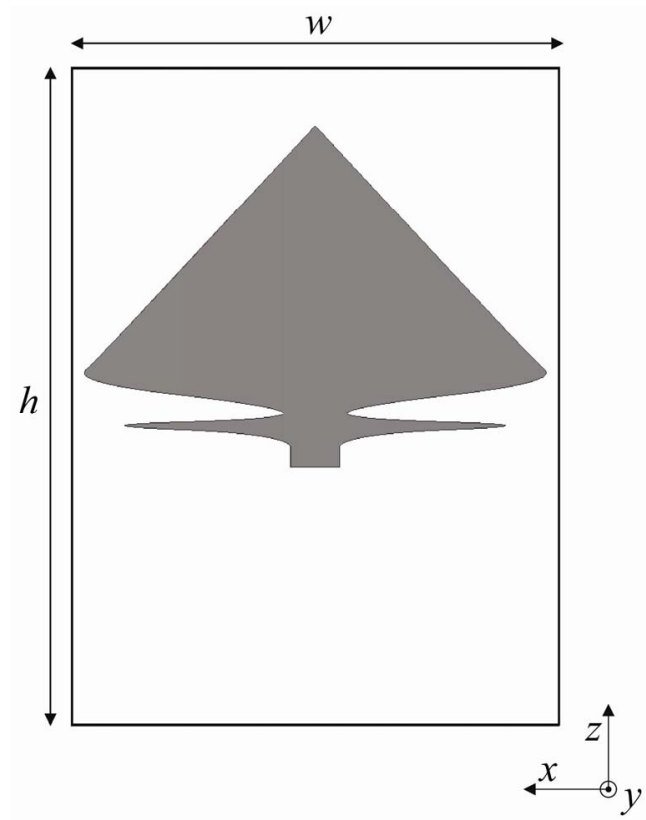

(a)

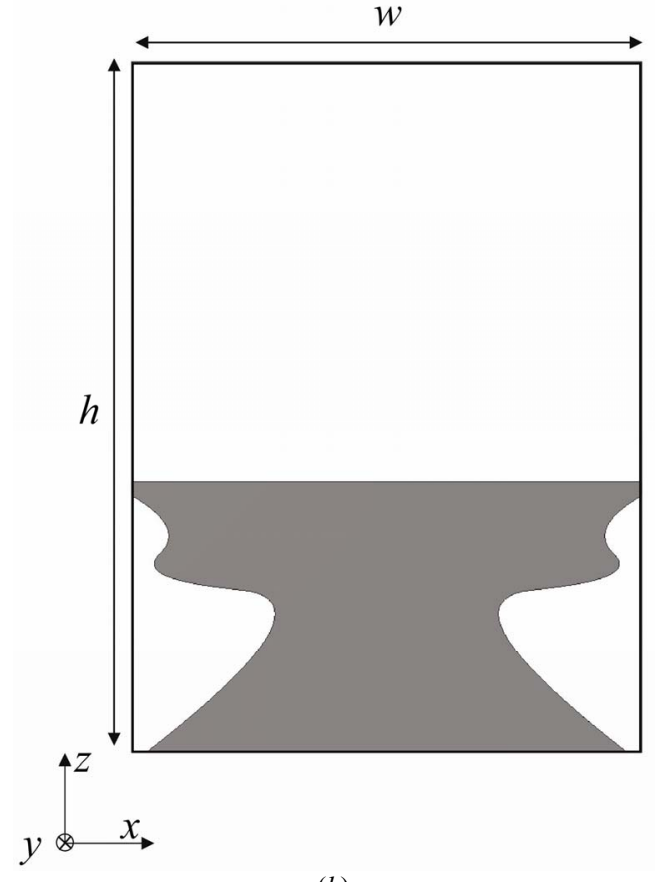

(b)

Fig. 1. Geometry of the optimized UWB spline element: $(a)$ front view (b) back view.

The overall antenna geometry is completely described by a set of $N$ geometric variables $\boldsymbol{\Omega}=\left\{\omega_{n} ; n=1, \ldots, N\right\}$, where $N=(K+$ $H)+J$, being $J$ the number of additional parameters describing the width $(w)$ and the height $(h)$ of the substrate, the width of the feeding point and the height of the ground plane.

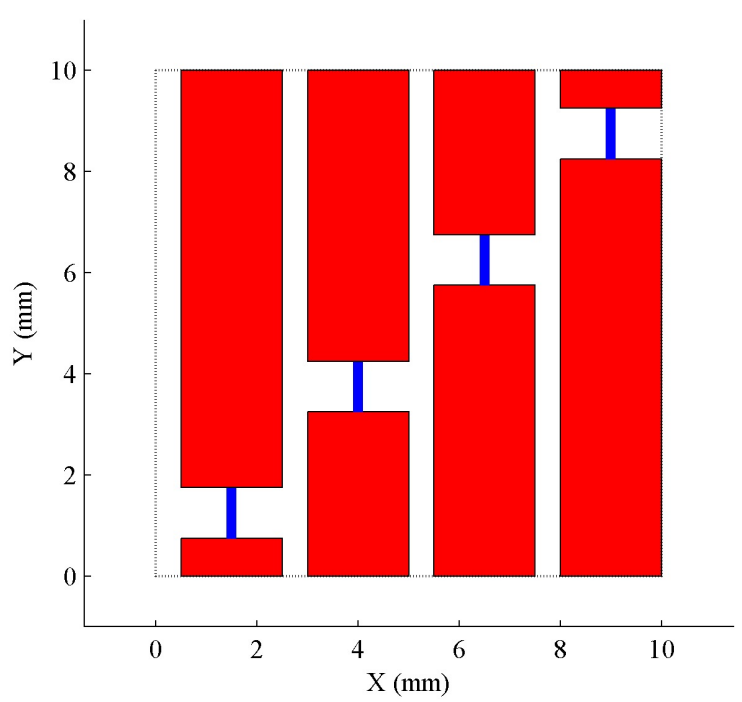

Fig. 2. Geometry of the AMC unit cell.

The synthesis problem is then formulated as finding the optimal set of parameters $\boldsymbol{\Omega}_{\mathrm{opt}}$ minimizing a given cost function $\Phi(\mathbf{\Omega})$

$$
\mathbf{\Omega}_{\text {opt }}=\arg \min _{\mathbf{\Omega}}[\Phi(\mathbf{\Omega})]
$$

where $\Phi(\mathbf{\Omega})=\alpha_{1} \Phi_{\mathrm{E}}(\boldsymbol{\Omega})+\alpha_{2} \Phi_{\mathrm{F}}(\boldsymbol{\Omega})+\alpha_{3} \Phi_{\mathrm{S}}(\mathbf{\Omega})$ and $\alpha_{i}, i=1, \ldots, 3$ are real weights $\alpha_{i} \in[0,1], i=1, \ldots, 3$. More precisely, $\Phi_{\mathrm{E}}(\boldsymbol{\Omega})$ is related to the antenna efficiency $E$, defined by the ratio between the energy of reflected $(w(t))$ and transmitted $(u(t))$ signals

$$
E=1-\int_{-\infty}^{+\infty}|w(t)|^{2} d t / \int_{-\infty}^{+\infty}|u(t)|^{2} d t
$$

$\Phi_{\mathrm{F}}(\boldsymbol{\Omega})$ is a term associated to the system fidelity $F$, defined as the maximum of the cross-correlation between transmitted and received signals

$$
F=\max _{\tau} \int_{-\infty}^{+\infty} \hat{v}(t-\tau) \hat{u}(t) d t
$$

where $\hat{u}(t)$ and $\hat{v}(t)$ are the normalized unitary energy versions of $u(t)$ and $v(t)$, respectively. Finally, $\Phi_{\mathrm{S}}(\boldsymbol{\Omega})$ is related to the antenna similarity factor $S$, which quantifies the omnidirectionality of the radiation pattern along a given plane 


$$
S(\vartheta, \varphi)=1-\frac{\int_{-\infty}^{+\infty}\left|\mathbf{e}_{r a d}\left(r, \vartheta_{0}, \varphi_{0}, t\right)-\mathbf{e}_{r a d}(r, \vartheta, \varphi, t)\right|^{2} d t}{\int_{-\infty}^{+\infty}\left|\mathbf{e}_{r a d}\left(r, \vartheta_{0}, \varphi_{0}, t\right)\right|^{2} d t}
$$

$\mathbf{e}_{r a d}(r, \theta, \varphi, t)$ being the radiated field and $\left(\theta_{0}, \varphi_{0}\right)$ the direction of maximum radiation.

In order to estimate the received signal waveform $v(t)$, a pair of identical antennas placed at a distance of $15[\mathrm{~cm}]$ apart is considered during the optimization process [22]. The final optimized antenna geometry is shown in Fig. 1. The optimized UWB spline antenna is characterized by an overall dimension of $47.5[\mathrm{~mm}] \times 64.5[\mathrm{~mm}]$ and shows a reflection coefficient $\left|\mathrm{S}_{11}\right| \leq-10[\mathrm{~dB}]$ within the frequency range 1.84-3.83 [GHz].

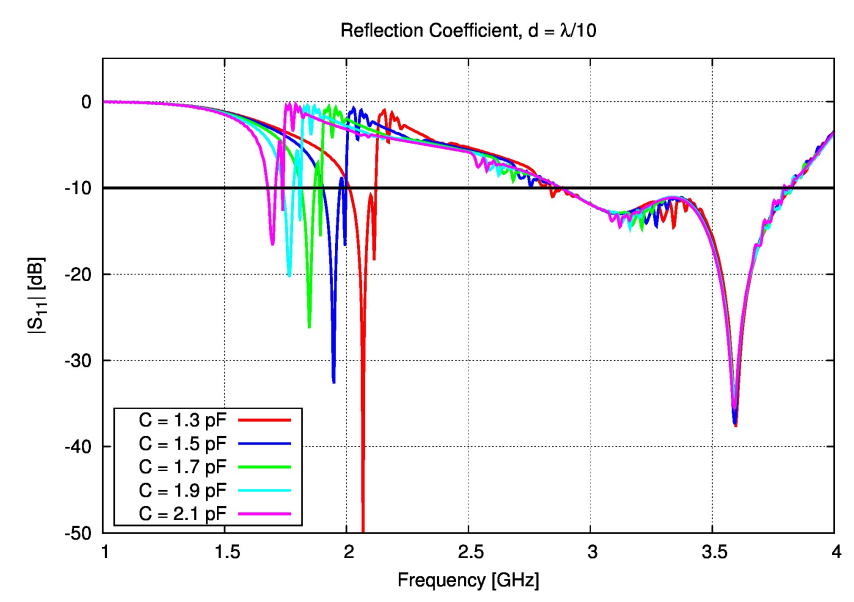

Fig. 3. Reflection coefficient of the UWB-on-AMC system, for different values of capacitance $C$.

\section{B. Mounting the UWB antenna on top of the tunable AMC}

The AMC unit cell is made of 8 metallic strips printed on a $10 \mathrm{x} 10\left[\mathrm{~mm}^{2}\right] R T D 5880$ substrate $\left(\varepsilon_{r}=2.2, \tan \delta=0.0008\right)$ of thickness $0.787[\mathrm{~mm}]$ [Fig. 2]. The metallic strips are connected by 4 tunable capacitors (blue lines in Fig. 2), whose capacitance $C$ can be adaptively modified to tune the AMC resonating frequency inside the 1-4 [GHz] bandwidth.

The UWB element is placed on top of a $15 \times 15$ periodic AMC groundplane, obtained by replicating in both $x$ and $y$ directions the unit cell, at a height of $d=\lambda_{\text {low }} / 10$, being $\lambda_{\text {low }}$ the free-space wavelength of the lowest operating frequency of the UWB antenna $\left(f_{\text {low }}=1.84[\mathrm{GHz}]\right)$.

\section{PRELIMINARY NUMERICAL RESUltS}

A set of numerical simulations is carried out, modifying the capacitance $C$ of the varactors of the AMC periodic surface. Fig. 3 shows the reflection coefficient measured at the input port of the UWB antenna when $C$ is chosen inside the range 1.3-2.1 [pF].
The resulting antenna system shows a tunable narrowband behavior of the return loss, with a controllable shift of the resonant frequency thanks to the reconfigurability of the tunable AMC groundplane.

\section{REFERENCES}

[1] A. Aminian, F. Yang and Y. Rahmat-Samii, "In-phase reflection and EM wave suppression characteristics of electromagnetic bandgap ground planes," IEEE Antennas and Propagation Society International Symposium, vol. 4, pp. 430-433, 2003.

[2] G. Goussetis, A. P. Feresidis, and J. C. Vardaxoglou, "Tailoring the AMC and EBG characteristics of periodic metallic arrays printed on grounded dielectric substrate," IEEE Trans. on Antennas and Propag., vol. 54, pp. 82-89, 2006.

[3] D. F. Sievenpiper, "Forward and backward leaky wave radiation with large effective aperture from an electronically tunable surface," IEEE Trans. on Antennas and Propag., vol. 53, no. 1, pp. 236-247, 2005.

[4] D. J. Kern, D. H. Werner, A. Monorchio, L. Lanuzza and M. J. Wilhelm, "The design synthesis of multiband artificial magnetic conductors using high impedance frequency selective surfaces," IEEE Trans. on Antennas and Propag., vol. 53, no. 1, pp. 8-17, 2005.

[5] J. R. Sohn, K. Y. Kim and H. S. Tae, "Comparative study on various artificial magnetic conductors for low-profile antenna," Progress in Electromagnetic Research, vol. 61, pp. 27-37, 2006.

[6] D. H. Werner and D.-H. Kwon, Eds., Transformation electromagnetics and metamaterials. London: Springer-Verlag, 2014.

[7] D. F. Sievenpiper, J. H. Schaffner, H. J. Song, R. Y. Loo and G. Tangonan, "Two-dimensional beam steering using an electrically tunable impedance surface," IEEE Trans. on Antennas and Propag., vol. 51, no. $10,2003$.

[8] L. Akhoondzadeh-Asl, D. J. Kern, P. S. Hall and D. H. Werner, "Wideband dipoles on electromagnetic bandgap ground planes," IEEE Trans. on Antennas and Propag., vol. 55, no. 9, pp. 2426-2434, 2007.

[9] F. Costa, A. Monorchio, S. Talarico and F. M. Valeri, "An active highimpedance surface for low-profile tunable and steerable antennas," IEEE Antenas and Wireless Propag. Lettters, vol. 7, pp. 676-680, 2008.

[10] M. G. Bray and D. H. Werner, "A broadband open-sleeve dipole antenna mounted above a tunable EBG AMC ground plane," Proc. IEEE APS Intern. Symposium, vol. 2, pp. 1147-1150, 2004.

[11] O. Luukkonen, C. R. Simovski, A. V. Raisanen and S. A. Tretyakov, "An efficient and simple analytical model for analysis of propagation properties in impedance waveguides," IEEE Trans. Microw. Theory Techn., vol. 56, no. 7, pp. 1624-1632, 2008.

[12] P. Rocca, M. Benedetti, M. Donelli, D. Franceschini, and A. Massa, "Evolutionary optimization as applied to inverse problems," Inverse Problems - 25 th Year Special Issue of Inverse Problems, Invited Topical Review, vol. 25, pp. 1-41, Dec. 2009.

[13] P. Rocca, G. Oliveri, and A. Massa, "Differential Evolution as applied to electromagnetics," IEEE Antennas Propag. Mag., vol. 53, no. 1, pp. 38-49, Feb. 2011

[14] L. Lizzi, F. Viani, R. Azaro and A. Massa, "A PSO-driven spline-based shaping approach for ultra-wideband (UWB) antenna synthesis," IEEE Trans. on Antennas and Propag., vol. 56, no. 8, pp. 2613-2612, 2008.

[15] L. Lizzi, R. Azaro, G. Oliveri, and A. Massa, "Printed UWB antenna operating over multiple mobile wireless standards," IEEE Antennas Wireless Propag. Lett., vol. 10, pp. 1429-1432, 2011.

[16] L. Lizzi, F. Viani, R. Azaro, and A. Massa, "Design of a miniaturized planar antenna for FCC-UWB communication systems," Microwave Opt. Technol. Lett., vol. 50, no. 7, pp. 1975-1978, Jul. 2008.

[17] F. Viani, L. Lizzi, R. Azaro, and A. Massa, "A miniaturized UWB antenna for wireless dongle devices," IEEE Antennas Wireless Propag. Lett., vol. 7, pp. 714-717, 2008.

[18] F. Viani, L. Lizzi, R. Azaro, and A. Massa, "Spline-shaped ultrawideband antenna operating in the ECC released frequency spectrum," Electronics Letters, vol. 44, no. 1, pp. 7-8, Jan. 2008. 
[19] L. Lizzi, F. Viani, R. Azaro, and A. Massa, "Optimization of a splineshaped UWB antenna by PSO," IEEE Antennas Wireless Propag. Lett., vol. 6, pp. 182-185, 2007.

[20] L. Lizzi, G. Oliveri, and A. Massa, "Planar monopole UWB antenna with UNII1/UNII2 WLAN-band notched characteristics," Progress in Electromagnetic Research B, vol. 25, pp. 277-292, 2010.

[21] L. Lizzi, F. Viani, and A. Massa, "Dual-band spline-shaped PCB antenna for Wi-Fi applications," IEEE Antennas Wireless Propag. Lett., vol. 8, pp. 616-619, 2009.
[22] L. Lizzi, G. Oliveri and A. Massa, "A time-domain approach to the synthesis of uwb antenna systems," Progress In Electromagnetic Research, vol. 122, pp. 557-575, 2012.

[23] L. Lizzi, G. Oliveri and A. Massa, "Exploitation of spline-based geometries for the time-domain synthesis of UWB antennas," Proc. 5th European Conf. Antennas Propagation (EuCAP), pp. 2382-2385, Rome, Italy, Apr. 11-15, 2011. 